\title{
FREQUENCY-DomaIn SYNTHETIC APERTURE FoCUSING TECHNIQUES FOR IMAGING WITH Single-ELEMENT FOCUSED TRANSDUCERS
}

\author{
Elyas Shaswary ${ }^{* 1,2}$, Jahan Tavakkoli ${ }^{\dagger 1,2}$ and Carl J. Kumaradas ${ }^{\ddagger 1}$ \\ ${ }^{1}$ Ryerson University, Toronto, Ontario, Canada. \\ ${ }^{2}$ Institute for Biomedical Engineering, Science and Technology, Toronto, ON, Canada.
}

\section{Introduction}

Synthetic aperture focusing techniques (SAFT) make the lateral spatial resolution of single-element conventional Bmode imaging more uniform, which leads to an improved spatial resolution and an extended depth of field. SAFT uses signal processing techniques to synthesize a larger aperture by moving a smaller physical aperture. Several SAFT algorithms have been proposed for different transducer geometries. For single-element focused transducer, the virtual point source techniques have proposed, which treats the geometric focus of the transducer as a virtual point source in the image reconstruction process [1].

In this work, we proposed two frequency-domain SAFT algorithms that are based on 2D matched filtering technique. The first algorithm is called virtual source FD-SAFT (FDSAFT-VS), which is similar to the algorithm presented in [2] that was implemented for flat circular transducers. However, FD-SAFT-VS treats the focal point of the transducer as a virtual flat circular source. The second algorithm is called deconvolution FD-SAFT (FD-SAFT$\mathrm{DE}$ ), which uses the simulated point-spread function (PSF) of the imaging system as a filter kernel for the matched filter in the image reconstruction.

\section{Frequency-domain virtual source SAFT (FD-SAFT-VS)}

The recorded echo signal can be expressed in frequencydomain as a convolution model as [2]:

$$
S\left(k_{x}, \omega\right)=\alpha \cdot A\left(k_{x}\right) \cdot \omega^{2} H(\omega) \cdot F\left(k_{x}, k_{z}\right),
$$

where $k_{x}$ and $k_{z}$ are wavenumbers in lateral and axial directions, respectively, $\omega$ is the angular frequency, $\alpha$ is a constant coefficient, $A\left(k_{x}\right)=\operatorname{jinc}^{2}\left(k_{x} a\right)$ is the directivity function for a flat circular transducer, $a$ is the radius of the transducer, $H(\omega)$ is the transmit and receive electromechanical impulse response of the transducer, and $F\left(k_{x}, k_{z}\right)$ is the object's reflectivity function.

The goal of FD-SAFT image reconstruction is to get a better representation of $F\left(k_{x}, k_{z}\right)$. FD-SAFT-VS splits the recorded data into pre-focal and post-focal regions and image reconstruction is carried out separately in each region. In the pre-focal region, the recorded data, the electro-mechanical impulse response and the excitation pulse are flipped in the axial direction prior to performing fast Fourier transform (FFT). However, in the post-focal region, the recorded data, the electro-mechanical impulse

\footnotetext{
*elyas.shaswary@ryerson.ca

jtavakkoli@ryerson.ca

tkumarad@ryerson.ca
}

response and the excitation pulse are transformed into Fourier domain without flipping. The image reconstruction is carried out in the pre-focal and post-focal regions as following:

$$
\begin{aligned}
\hat{F}\left(k_{x}, k_{z}\right)=\mathcal{S}^{-1} & \left\{\exp \left[j\left(\sqrt{4 k^{2}-k_{x}^{2}}-2 k\right) z_{c}\right] \cdot A^{*}\left(k_{x}\right)\right. \\
& \left.\cdot \omega^{2} H^{*}(\omega) \cdot P^{*}(\omega) \cdot S\left(k_{x}, \omega\right)\right\},
\end{aligned}
$$

where $z_{c}$ is the perpendicular distance from the transducer to the midpoint of the ROI, $k$ is the wavenumber, and the asterisk represents complex conjugate. $\mathcal{S}^{-1}\{\cdot\}$ is the Stolt transformation, which transforms $\left(k_{x}, \omega\right)$ into $\left(k_{x}, k_{z}\right)$ coordinates defined by: $k_{z}\left(k_{x}, \omega\right)=\sqrt{4 k^{2}-k_{x}}$ (for details see [3]). Finally, inverse FFT is performed to pre-focal and post-focal regions and then pre-focal region is flipped in the axial direction to its original orientation before joining it with the post-focal region to get the final reconstructed image.

\section{Frequency-domain deconvolution SAFT (FD-SAFT-DE)}

If the ultrasound image formation is assumed to be based on the convolution model, then an image, $S\left(k_{x}, \omega\right)$, can be modelled as a convolution of the system's PSF, $\operatorname{PSF}\left(k_{x}, \omega\right)$, and object's reflectivity function, $F\left(k_{x}, k_{z}\right)$ in frequencydomain as following:

$$
S\left(k_{x}, \omega\right)=F\left(k_{x}, k_{z}\right) \cdot \operatorname{PSF}\left(k_{x}, \omega\right) .
$$

The PSF represents the response of the system to a point scatterer and it takes into account all the characteristics of the imaging system. However, the PSF depends on the position between the transducer and point scatterer. For a single-element focused transducer, the PSF at the focal point of the transducer is distorted the least because the diffraction effect at that position is minimal.

FD-SAFT-DE performs the image reconstruction by deconvolving the simulated PSF of the system from the recorded echo data via matched filtering, and then followed by Stolt transformation, as:

$$
\hat{F}\left(k_{x}, k_{z}\right)=\mathcal{S}^{-1}\left\{P S F^{*}\left(k_{x}, \omega\right) \cdot S\left(k_{x}, \omega\right)\right\}
$$

\section{Method}

The performances of the proposed algorithms were evaluated using simulated radio-frequency (RF) data. The simulated data were generated using Field II simulation software [4] and the simulated transducer was a commercially-available single-element spherically focused with $25 \mathrm{MHz}$ central frequency, $7 \mathrm{~mm}$ diameter and $15 \mathrm{~mm}$ 
focal length (RMV-710B, FUJFILM VisualSonics Inc., Toronto, Canada). The electro-mechanical impulse response of the transducer was determined experimentally. The values of the simulation parameters are shown in Table 1. The medium consisted of water with several point scatterers at different axial distances. White Gaussian noise, mimicking electronic noise, was added to the generated RF data, where its magnitude and spectrum were set to match the RF data that was recorded experimentally. The performances of the proposed algorithms were compared to the conventional B-mode. The contour area of each point scatterer from the reconstructed images was measured at -6 $\mathrm{dB}$ for the quantification of the spatial resolution and at -18 $\mathrm{dB}$ for the quantification of the side lobes. Electronic signalto-noise ratio (SNRe) of each point scatterer was also measured similar to ref. [5].

Table 1: Values of the simulation parameters.

\begin{tabular}{ll}
\hline Parameter & Value \\
\hline Speed of sound & $1540 \mathrm{~m} / \mathrm{s}$ \\
\hline Attenuation & $0.0022 \mathrm{~dB} /(\mathrm{cm} . \mathrm{MHz})$ \\
\hline Sampling frequency & $420 \mathrm{MHz}$ \\
\hline Excitation pulse & 1 cycle sinusoidal wave \\
\hline
\end{tabular}

\section{Results}

The reconstructed images of the conventional B-mode, FDSAFT-VS and FD-SAFT-DE of the point scatterer in water are shown in Figure 1. The measurement of contour area at $6 \mathrm{~dB}$ and $-18 \mathrm{~dB}$ levels, and SNRe of each point scatterer from the reconstructed images are shown in Figure 2.

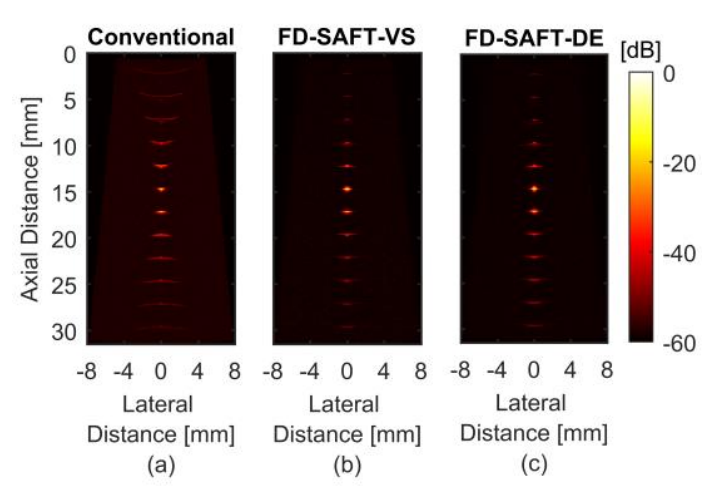

Figure 1: The reconstructed images of the point scatterer phantom in water. The conventional B-mode (a), FD-SAFT-VS (b), and FDSAFT-DE (c) are shown.

\section{Discussion}

As shown in Figure 2, the conventional B-mode had the largest areas of the contour at both $-6 \mathrm{~dB}$ and $-18 \mathrm{~dB}$ and the contour areas decreased around the focal distance of $15 \mathrm{~mm}$. FD-SAFT-DE had smaller contour areas compared to the conventional B-mode. In addition, FD-SAFT-VS had generally the smallest contour areas. Thus, both SAFT algorithms were able to extend the depth of field beyond the conventional B-mode. The reason for these improvements is that the FD-SAFT-VS and FD-SAFT-DE compensate for the diffraction effects, the electro-mechanical impulse response and the excitation pulse of the transducer. Furthermore, the SNRes of all methods were highest at the focal distance and they decreased away from the focal distance. FD-SAFT-VS and FD-SAFT-DE had higher SNRes compared to the conventional B-mode by an average of $3.62 \mathrm{~dB}$ and $5.35 \mathrm{~dB}$, respectively.

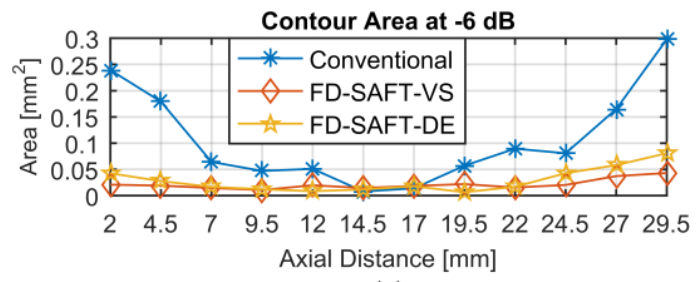

(a)

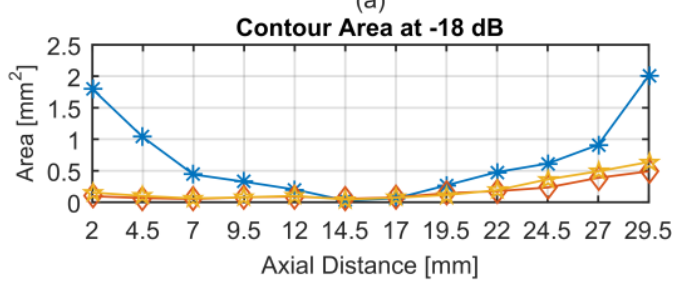

(b)

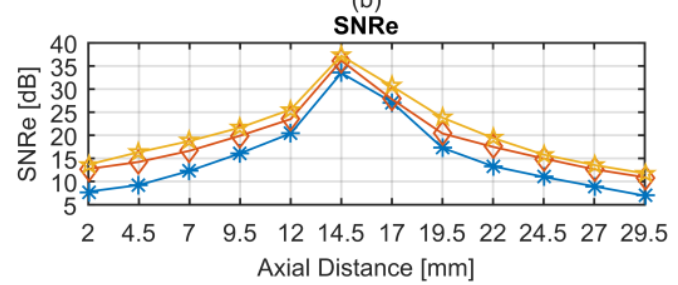

(c)

Figure 2: The measurements of the contour area at $-6 \mathrm{~dB}$ (a) and $18 \mathrm{~dB}(\mathrm{~b})$, and measurement of the SNRe (c).

\section{Conclusion}

Among the methods studied, the FD-SAFT-VS had the smallest spatial resolution and the FD-SAFT-DE had the second smallest spatial resolution. In addition, the FDSAFT-DE generally had the higher SNRe compared to other methods. Thus, the proposed methods made the spatial resolution more uniform and extended the depth of field of conventional B-mode ultrasound imaging.

\section{References}

[1] C. Passmann and H. Ermert, "A 100-MHz ultrasound imaging system for dermatologic and ophthalmologic diagnostics," IEEE Trans. Ultras. Ferroe. Freq. Control, 43(4), 545-552, 1996.

[2] T. Stepinski, "An implementation of synthetic aperture focusing technique in frequency domain.," IEEE Trans. Ultras. Ferroe. Freq. Control, 54(7), 1399-408, 2007.

[3] M. Soumekh, Synthetic aperture radar signal processing with MATLAB algorithms. New Jersey: John Wiley \& Sons, Inc., 1999.

[4] J. A. Jensen, "Field: a program for simulating ultrasound systems," Med. Biol. Eng. Comp., 34(1), 351-353, 1996.

[5] M. Karaman, P.-C. Li, and M. O'Donnell, "Synthetic aperture imaging for small scale systems," IEEE Trans. Ultras. Ferroe. Freq. Control, 42(3), 429-442, 1995. 